Review

\title{
Heart rate variability in individuals with Down syndrome - A systematic review and meta-analysis
}

\author{
Tatiana Dias de Carvalho ${ }^{\mathrm{a}, \mathrm{b}, *}$, Thais Massettic ${ }^{\mathrm{c}}$,Talita Dias da Silva ${ }^{\mathrm{a}}$, Tânia Brusque Crocetta ${ }^{\mathrm{d}}$, \\ Regiani Guarnieri ${ }^{\mathrm{d}}$, Luiz Carlos Marques Vanderlei ${ }^{\mathrm{e}}$, Carlos Bandeira de Mello Monteiro ${ }^{\mathrm{c}}$, \\ David M. Garner ${ }^{\mathrm{f}}$, Celso Ferreira ${ }^{\mathrm{a}}$ \\ ${ }^{a}$ Universidade Federal de São Paulo (UNIFESP), Departamento de Medicina, Disciplina de Cardiologia, São Paulo, SP, Brazil \\ b Universidad Nacional de La Matanza (UNLaM), Departamento de Ciencias de la Salud, Kinesiología y Fisiatría, San Justo, BA, Argentina \\ ${ }^{\mathrm{c}}$ Universidade de São Paulo (USP), Faculdade de Medicina, Programa de Pós-graduação Ciências da Reabilitação, São Paulo, SP, Brazil \\ ${ }^{\mathrm{d}}$ Faculdade de Medicina do ABC (FMABC), Laboratório de Escrita Científica, Santo André, SP, Brazil \\ e Universidade Estadual Paulista (UNESP), Departamento de Fisioterapia da Faculdade de Ciências e Tecnologia, Presidente Prudente, SP, Brazil \\ ${ }^{\mathrm{f}}$ Cardiorespiratory Research Group, Department of Biological and Medical Sciences, Oxford Brookes University, Headington Campus, Gipsy Lane, Oxford OX3 OBP, United \\ Kingdom
}

\section{A R T I C L E I N F O}

\section{Keywords:}

"Autonomic nervous system"

"Cardiac autonomic modulation"

"Down syndrome"

"Meta-analysis"

"Systematic review"

\begin{abstract}
A B S T R A C T
Introduction: Down syndrome (DS) results in many changes, including dysfunction in cardiac autonomic modulation. Heart rate variability (HRV) analysis evaluates the autonomic function and it is a predictor of adverse cardiovascular events.

Objective: To present results of a systematic review and a meta-analysis about heart rate variability in individuals with DS.

Method: A systematic review was performed on PubMed, PubMed Central and Web of science databases. We included articles that exhibited all the terms: "Down Syndrome", "heart rate variability", "autonomic nervous system", "autonomic dysfunction" and "cardiac autonomic modulation". We conducted the meta-analysis to compare "DS" to "controls" during rest. Random effects models were used, as were appropriate tests for heterogeneity.

Results: From 271 studies, 13 were included in our review. These are conducted with volunteers from a wide age range, of either gender, and not taking medications. Meta-analysis displayed that there were no significant differences between the groups at rest, except the RMSSD, which revealed a significant $(\mathrm{Z}=-2.80, p=0.005)$ main effect (Hedge's $g=-0.55,95 \%$ CI $[-0.93 ;-0.16]$ ), indicating difference in individuals with DS compared with controls.

Conclusion: There is autonomic dysfunction in individuals with DS, which may or may not be expressed at rest, but it is usually demonstrated in an autonomic task. Meta-analysis specified that there was no significant alteration between DS and the controls during rest, except RMSSD index which was lower in DS than controls. PROSPERO: CRD42017068647.
\end{abstract}

\section{Introduction}

Down syndrome (DS) is the most frequently occurring chromosomal abnormality in humans (trisomy of whole or part of chromosome 21), affecting about one in every 750 live births in all populations (Kazemi et al., 2016; Kazemi et al., 2017). Frequently, individuals with DS present muscle hypotonia, hypothyroidism, gastrointestinal and pulmonary disorders, leukemia, delayed psychomotor and neurological development, audio vestibular and visual impairment, early-onset
Alzheimer's disease, dementia, and congenital heart disease (Van Gameren-Oosterom et al., 2012; Fernhall et al., 2013).

Current studies indicate that individuals with DS exhibit a dysfunction in autonomic cardiac modulation, when compared with nondisabled control subjects. Overall, individuals with DS have low physical work capacity, chronotropic incompetence and significantly reduced heart rate and blood pressure responses to autonomic tasks, such as exercise and the tilt test (Iellamo et al., 2005; Fernhall et al., 2013; Bunsawat et al., 2015). According to Fernhall and Otterstetter (2003)

\footnotetext{
* Corresponding author at: Universidade Federal de São Paulo (UNIFESP), Rua Napoleão de Barros, 715 Térreo Vila Clementino, São Paulo, SP, Brazil.

E-mail address: carvalho.td1@gmail.com (T.D.d. Carvalho).
} 
the dysfunction in autonomic cardiac modulation in DS could be related to depressed sympathetic tone or a response of incomplete vagal withdrawal.

Under typical conditions, the chronotropic state of the heart is entirely regulated by the sinoatrial (SA) node, which is directly innervated by the autonomic nervous system (ANS) that can be split into two efferents; parasympathetic (vagal) and sympathetic (phrenic) (Draghici and Taylor, 2016). Adjustments in at least one of those efferents can be considered an autonomic dysfunction, which may represent an important adverse factor, since the autonomic functioning controls part of the internal functions of the body and, it can be associated with increased risk of early mortality and morbidity (Task Force, 1996; Baynard et al., 2004; Angiovlasitis et al., 2011).

One of the ways to assess the ANS is heart rate variability (HRV), which is a simple, inexpensive and noninvasive measure of the balance between sympathetic and parasympathetic mediators of heart rate (Karim et al., 2011; Draghici and Taylor, 2016). It defines the fluctuation of the intervals between consecutive heart beats (RR intervals) and these are related to the stimuli of the ANS on the SA node (Task Force, 1996; Vanderlei et al., 2009).

HRV offers an important index as a potential marker of physiological stress and health for organism functions associated with adaptability and health (Draghici and Taylor, 2016). It has been considered a predictor of adverse cardiovascular events in different conditions (Task Force, 1996; Vanderlei et al., 2009; Karim et al., 2011). Understanding these topics specifically in DS can further information about the influence of this syndrome in the ANS function and provide support to improve therapies in order to enhance the quality of life of these individuals. To the best of our knowledge, there is neither meta-analysis nor revision undertaken jointly in this manner.

Considering the above interpretations, the purpose of this study is to present results of a systematic review and a meta-analysis about heart rate variability in individuals with DS.

\section{Methods}

This review was completed according to the Preferred Reporting Items for Systematic Reviews and Meta-Analyses (PRISMA) guidelines, so providing a comprehensive framework which accurately assesses indicators of quality and risk of biases of included studies (Hutton et al., 2015). The protocol for this review was previously registered with PROSPERO, number registration: CRD42017068647.

\subsection{Eligibility criteria}

At the outset, the titles of articles were evaluated, and then their abstracts were screened, according to some conditions. Articles included: (1) a diagnosis of Down syndrome, (2) HRV analysis, (3) studies that included autonomic nervous system, (4) studies that aimed to study cardiac autonomic modulation. Articles were excluded if they were: (1) not data-based (e.g. books, theoretical papers, or secondary reviews), (2) not written in the English language, (3) had populations not explicitly identified as having a diagnosis of DS, or (4) did not include HRV analysis.

\subsection{Information sources and search}

This appraisal was based on a systematic search of published articles available through July 2017. The article search was performed in Medline/PubMed, PubMed Central- PMC, and Web of Sciences databases - WOS, through keywords that must be in all fields (Table 1).

In brief, reference lists of retrieved studies were comprehensively searched for with additional relevant studies (Arab et al., 2016). Keywords and combinations of keywords were used to search the electronic databases and were organized following the Population Intervention Comparison Outcome (PICO) model. All identified studies were

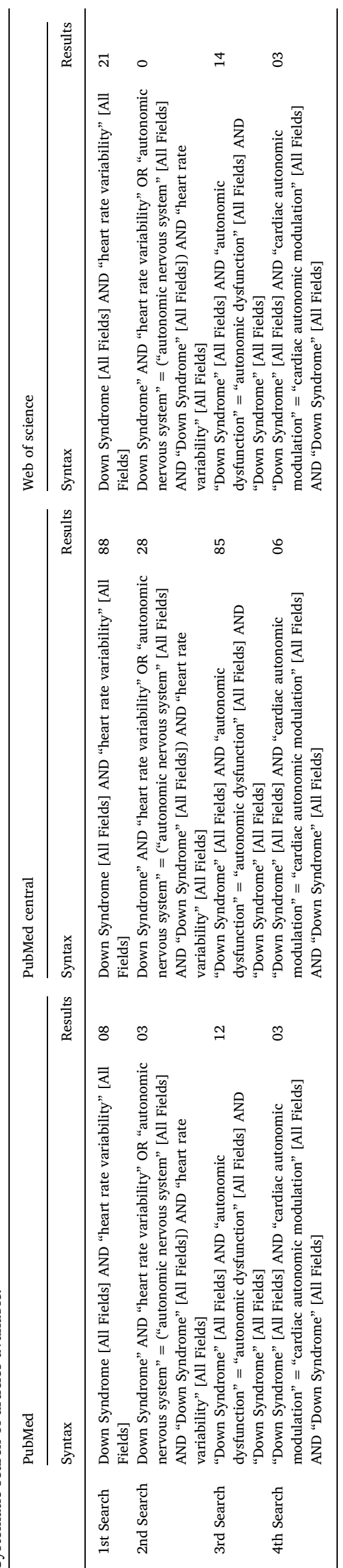




\section{PICOS Population: Down Syndrome}

Intervention: HRV evaluation, with or without autonomic tasks

Comparator (control): individuals without DS

Outcome: altered HRV indexes

Study design(s): Clinical trials, case-control, cross-sectional, case

reports and case series

Fig. 1. P.I.C.O.S.

collected in EndNote Web (Thomson Reuters) and all duplicates were removed. Fig. 1 illustrates the search strategy.

To select the articles, we imposed four stages: (1) seeking articles in databases and reading the titles and abstracts; (2) exclusion of works by analysis of title, abstract, and other inclusion criteria; (3) full-text analysis of findings within eligible articles (16-18) and (4) in order not to misplace any articles that are relevant to the results, we surveyed the reference section of selected articles.

\subsection{Study selection and data collection processes}

After undertaking the initial literature searches, each study title and abstract was screened for eligibility according to the inclusion criteria by the consensus of at least three authors (TDC, TM, TBC). Full texts of all theoretically relevant studies were subsequently retrieved and further examined for eligibility. The PRISMA flow diagram (Fig. 1) provided more detailed information regarding the selection process of studies.

To increase confidence in the choice of articles, two researchers (TDC and TM) reviewed all potentially relevant articles independently. After reading all of these, the researchers came to an agreement as to which articles fulfilled the inclusion criteria (Massetti et al., 2014; Menezes et al., 2015; Massetti et al., 2016).

\subsection{Quality and risk of bias in individual studies}

As in the Arab et al. (2016) study, we surveyed GRADE's Working Group on Recommendation, Development and Evaluation to give a rationale for inclusion of studies, the main element considered in the strength of evidence was the study design, generally categorized as observational (low evidence) and randomized trials (high evidence). The quality of the study (detailed study methods and execution) and the presence of several limitations were likewise considered in evaluation of the strength-analysis of the evidence.

\subsection{Data analysis}

We conducted the meta-analysis using $\mathrm{R}$ software (version 3.1.2, $\mathrm{R}$ Foundation for Statistical Computing, Vienna, Austria) to compare "Down Syndrome group" to "control group" subjects for studies that presented absolute values of mean and standard deviation (mean \pm S.D.) using "Standard mean differences" (SMD) $\pm 95 \%$ confidence intervals (CI). Random effect models were imposed, as were appropriate tests for heterogeneity (R Core Team, 2017).

Due to the difficulties of analysis and scarce utilization in the results section of the nonlinear data (Angiovlasitis et al., 2011), we chose to undertake the meta-analysis only with linear HRV indexes, comparing DS and control groups during the resting state. In addition to RRi (RR intervals in milliseconds, ms), we studied in the time domain the indexes SDNN (mean standard deviation of all normal RR intervals, ms) and RMSSD (square root of the mean of squared differences between successive beat intervals, $\mathrm{ms}$ ). In the frequency domain, we analyzed LF (low frequency, $\mathrm{ms}^{2}$ and normalized units, nu), HF (high frequency, $\mathrm{ms}^{2}$ and $\mathrm{nu}$ ) and LF/HF ratio. RMSSD and HF indexes denoting the parasympathetic ANS, and SDNN and LF indexes represent the overall modulation with sympathetic ANS dominance (Vanderlei et al., 2009; Arab et al., 2016; Draghici and Taylor, 2016).

\subsection{Synthesis of results}

Meta-analyses of experimental outcomes, including the calculation of weighted mean effect sizes, $95 \%$ confidence intervals, $\mathrm{I}^{2}$ heterogeneity values, and $p$-values using the random effects model were performed with the metafor package in $\mathrm{R}$ (Viechtbauer and Viechtbauer, 2016). All error bars in the forest plots are 95\% confidence intervals. Forest plots were created with metafor and custom R scripts. Meta-analysis was permitted through a random effects model for the separate analysis of each of the two outcome sets (time domain and frequency domain). Potential publication bias was investigated by visual inspection of funnel plots of effect size and standard error (Peschel et al., 2016).

\subsection{Role of the funding source}

The benefactors of the study had no part in study design, data collection, data analysis, data interpretation, writing of the report, or the decision to submit for publication. All authors had full access to all data in the study.

\section{Results}

\subsection{Study selection}

This review identified 271 studies in the databases (Medline/ PubMed $=26$, PubMed Central $=207$, and Web of Sciences databases $=38$ ), using a cross between keywords. The screening phase involved the exclusion of duplicate articles, those that were not original articles, and those that were not written in the English language, resulting in the exclusion of 237 studies.

Consequently, 34 studies were screened for eligibility through a review of their titles, abstracts and full texts. Of these studies, 21 were excluded for the following explanations: unrelated topic, no analysis of HRV indexes and unavailable text. So, 13 empirical studies fully met the aforesaid eligibility criteria for inclusion in the systematic review process (Fig. 2).

Tables 2 and 3 present the main information of the selected articles. 


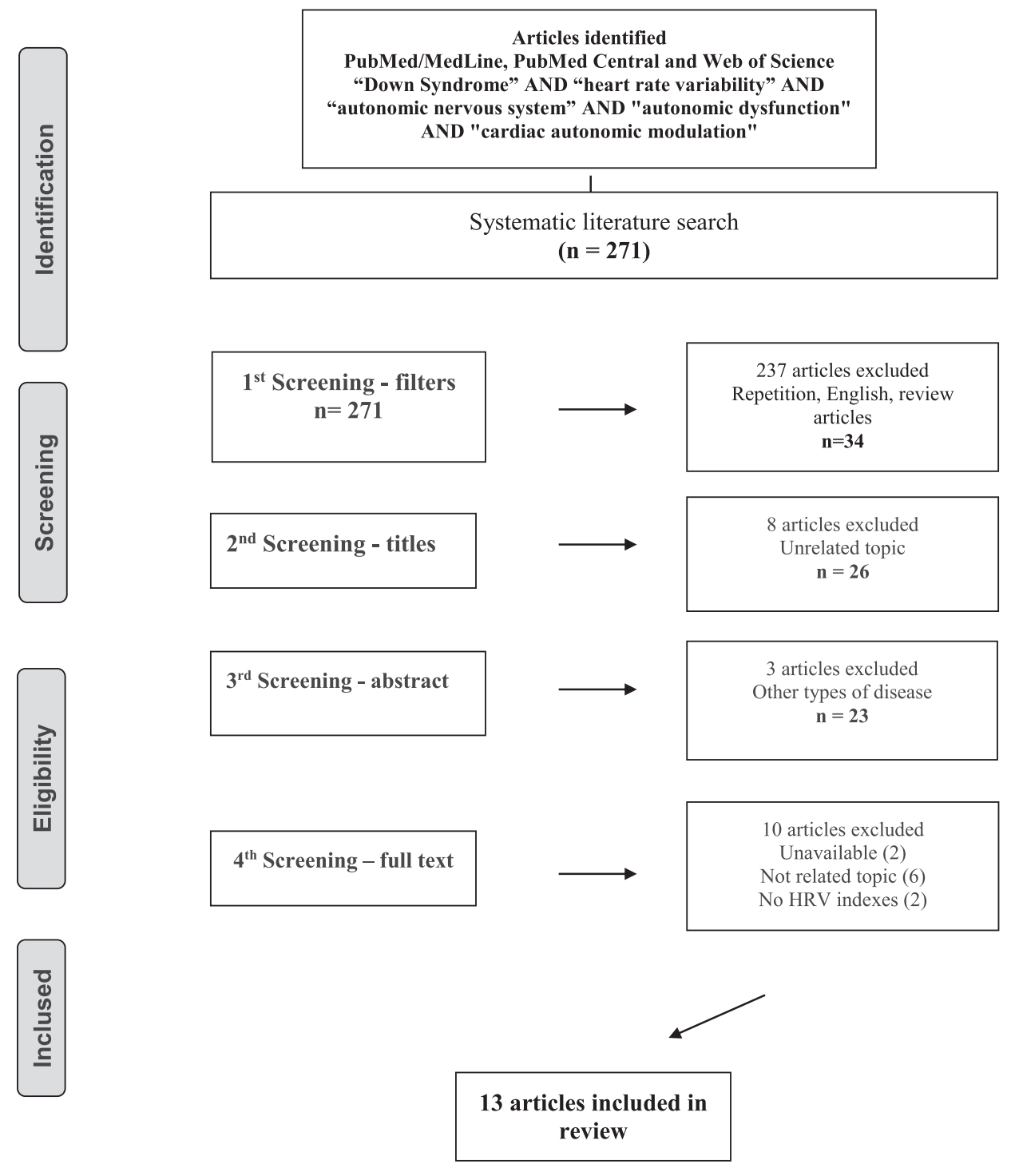

Fig. 2. Procedures for determination of eligibility.

Adapted from Moher et al. (2009).

\subsection{Meta-analysis}

Meta-analyses were computed discretely in the time and frequency domains. Data presented in tables and graphs were extracted. The measurements obtained through the graphs are indicated in the figures with "g" superscript. The studies excluded from the meta-analyses were presented with the following motivations: a) Baynard et al. (2004) because the control group was comprised of subjects with mental retardation without Down syndrome; b) Iellamo et al. (2005) because the data were presented as median and the correspondent author replied that they did not have the original data; c) Bunsawat et al. (2015) and Figueiroa Figueroa et al. (2005) because the participants are essentially the same as in Bunsawat and Baynard (2016), and we decided to use the most recent.

\subsubsection{Mean $R R$}

Angiovlasitis et al. (2011) did not discuss the Mean and SD. Similarly, the datasets were obtained from the figure before analysis.

Random effects analysis on the studies that included Mean RR outcomes exhibited a weighted average effect size of -0.37 (95\% CI $[-0.81,0.07], p=0.10)$, thus showing no significant difference in patients with DS $(n=41)$ compared with controls $(n=41)$. Results are displayed in Fig. 3(a). Heterogeneity was low $\left(\mathrm{I}^{2}=0 \% ; \mathrm{H}^{2}=1.0\right)$.

\subsubsection{Time domain - SDNN}

Random effects analysis on the studies that included SDNN outcomes $(n=3)$ showed a weighted average effect size of $-0.24(95 \% \mathrm{CI}$ $[-1.08,0.61], p=0.58)$, thus showing no significant difference in SDNN of patients with DS $(n=85)$ compared with controls $(n=59)$. Results are shown in Fig. 3(b). Heterogeneity was high $\left(\mathrm{I}^{2}=81 \%\right.$; $\left.\mathrm{H}^{2}=5.34\right)$.

\subsubsection{Time domain - RMSS}

Effect sizes (i.e. standardized mean differences between DS and controls) of all included studies are presented in Fig. 3(c). Random effect meta-analysis across all included studies $(n=4)$ revealed a significant $(Z=-2.80, p=0.005)$ main effect (Hedge's $g=-0.55,95 \%$ CI $[-0.93 ;-0.16])$, indicating difference in patients with DS $(n=94)$ compared to controls $(n=68)$. No significant heterogeneity was observed $\left(\mathrm{Tau}^{2}=0.19, \mathrm{Chi}^{2}=3.56, \mathrm{df}=3, P=0.31, \mathrm{I}^{2}=22 \%\right)$. Furthermore, visual examination of the funnel plot (Fig. 2(c)) revealed no outliers demonstrating no publication bias (Rank Correlation Test for Funnel Plot Asymmetry, Kendall's tau $=-0.67, p=0.33$ ). 


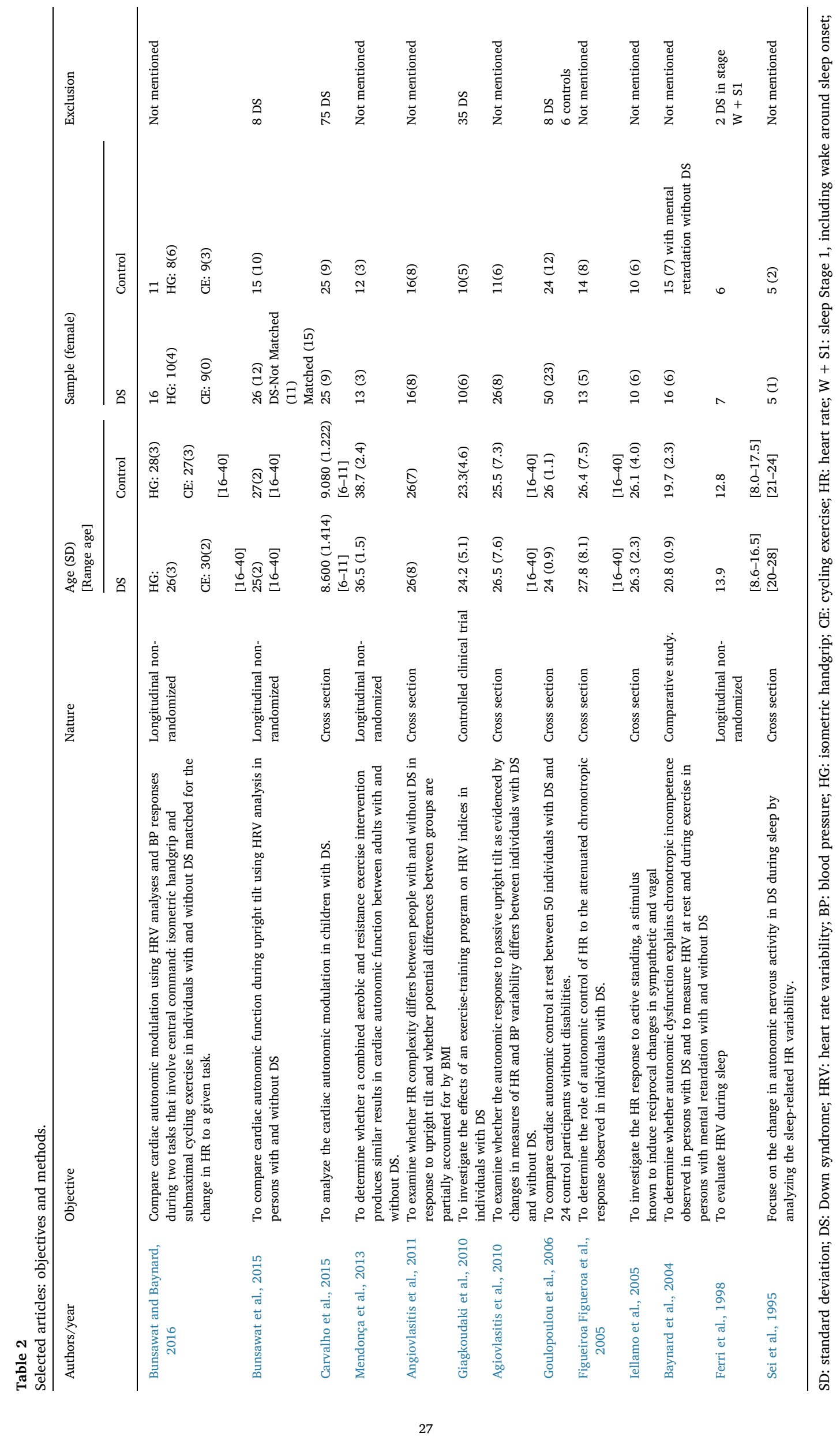


Table 3

Selected articles: protocols and main outcomes.

\begin{tabular}{|c|c|c|}
\hline Authors/year & $\begin{array}{l}\text { Protocol } \\
\text { HRV analysis } \\
\text { Instrument } \\
\text { Record time }\end{array}$ & Main outcomes \\
\hline
\end{tabular}

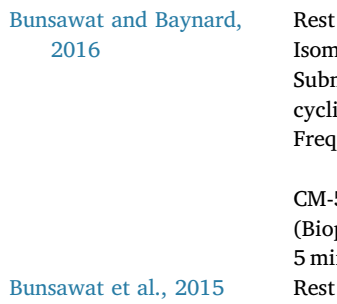
Supine rest and head-up tilt at an angle of 80 degrees

Frequency domain

Finger photo-plethysmography (Portapres, TNO Biomedical Instrumentation, Amsterdam, The Netherlands)

5 min of rest

5 min of upright tilt

Goulopoulou et al., 2006 Rest in the supine position and Treadmill exercise test

Frequency and time domains

Modified CM5 ECG lead, interfaced with data collection and interpretation software (Biopac Systems, CA)

$5 \mathrm{~min}$ of rest
Figueiroa Figueroa et al. 2005
With normal HR responses to these tasks, this subset of individuals with DS exhibited normal autonomic responses to HG, despite an overall lower sympathovagal balance.

Cardiac autonomic and BP responses are not uniform in individuals with DS.

Reduced sympathetic dominance in response to passive upright tilt in persons with DS despite similar HR responses.

Results indicate increasing indices representing the sympathetic branch of the ANS and those that indicate the overall modulation in children with DS.

Exercise training intervention enhanced the HFnu and decreased the LFnu of participants with and without DS at rest in the supine position.

People with DS show smaller decrease in HR complexity in response to upright tilt than people without DS. This response is due to the higher BMI of people with DS. Resting HR complexity does not differ between persons with and without DS.

Specifically, exercise training was able to restore vagal modulation and improve sympathovagal balance to levels seen in healthy persons without DS.

Individuals with DS show less vagal withdrawal and a smaller increase in sympathetic modulation in response to upright tilt than individuals without

Total HRV in the time domain was reduced at rest in individuals with Down syndrome when compared to their nondisabled peers, manifesting possible autonomic dysfunction in this population.

Attenuated HR and SBP responses to handgrip exercise in individuals with DS are due to blunted vagal withdrawal. 
Table 3 (continued)

\begin{tabular}{|c|c|c|}
\hline Authors/year & $\begin{array}{l}\text { Protocol } \\
\text { HRV analysis } \\
\text { Instrument } \\
\text { Record time }\end{array}$ & Main outcomes \\
\hline & $\begin{array}{l}\text { Rest, handgrip and recovery } \\
\text { Frequency domain } \\
\text { One lead ECG interfaced with a computer data acquisition system } \\
\text { (BIOPAC, Santa Barbara, CA). }\end{array}$ & \\
\hline Iellamo et al., 2005 & $\begin{array}{l}2 \text { min period at rest, handgrip and recovery. } \\
\text { Supine rest and active orthostatism. } \\
\text { Frequency domain }\end{array}$ & $\begin{array}{l}\text { Blunted sympathetic activation and vagal withdrawal associated with a lesser } \\
\text { reduction in baroreflex opposition to HR changes in response to active } \\
\text { orthostatism in this patient population. }\end{array}$ \\
\hline & $\begin{array}{l}\text { Electrocardiographic signal was recorded from a precordial chest } \\
\text { lead (Biopac Systems). }\end{array}$ & \\
\hline \multirow[t]{2}{*}{ Baynard et al., 2004} & $\begin{array}{l}10 \text { min of supine rest followed by } 10 \mathrm{~min} \text { of active orthostatism. } \\
\text { Rest } \\
\text { Submaximal exercise }\end{array}$ & \multirow[t]{2}{*}{$\begin{array}{l}\text { Greater parasympathetic activity on the SA node at rest in participants with DS } \\
\text { than in their peers with mental retardation without DS. Both groups exhibited } \\
\text { similar levels of parasympathetic withdrawal during submaximal exercise. }\end{array}$} \\
\hline & $\begin{array}{l}\text { Wireless heart rate monitor } \\
5 \text { min of rest } \\
4 \text { min submaximal exercise }\end{array}$ & \\
\hline \multirow[t]{2}{*}{ Ferri et al., 1998} & Sleep stages & $\begin{array}{l}\text { Brainstem dysfunction in DS, responsible for the abnormal imbalance between } \\
\text { the sympathetic (increased) and vagal (decreased) systems. }\end{array}$ \\
\hline & $\begin{array}{l}\text { Frequency and time domains } \\
\text { ECG } \\
\text { Oxford MPA-II recorder }\end{array}$ & \\
\hline \multirow[t]{3}{*}{ Sei et al., 1995} & $\begin{array}{l}10 \text {-min period in each sleep stages } \\
\text { Sleep stages }\end{array}$ & $\begin{array}{l}\text { Results indicate that parasympathetic tone increases, and sympathetic tone } \\
\text { decreases in the process from wakefulness to slow-wave sleep. }\end{array}$ \\
\hline & $\begin{array}{l}\text { Frequency domain } \\
\text { ECG da polisonografia }\end{array}$ & \\
\hline & 10 -min period in the sleep stages & \\
\hline
\end{tabular}

DS: Down syndrome; HRV: heart rate variability; BP: blood pressure; HG: isometric handgrip; CE: cycling exercise; HR: heart rate; SBP: systolic blood pressure; ECG: electrocardiogram;

\subsubsection{Frequency domain - LFms ${ }^{2}$}

There was no significant difference in LFms ${ }^{2}$ between the DS subjects and control group at rest, with outcomes showing a weighted average effect size of -0.87 (95\% CI [ $-2.28,0.53], p=0.22)$. Results are shown in Fig. 3(d). Heterogeneity was high $\left(\mathrm{I}^{2}=94 \% ; \mathrm{H}^{2}=16.23\right)$.

Assessment for publication bias obtained by the Rank Correlation Test for Funnel Plot Asymmetry and the visual inspection suggested publication bias for $\mathrm{LFms}^{2}$ (also Kendall's tau was insignificant, -0.40 , $p=0.48)$.

\subsubsection{Frequency domain $-\mathrm{HFms}^{2}$}

There was no significant difference in $\mathrm{HFms}^{2}$ between the DS subjects and control group at rest, with outcomes displaying a weighted average effect size of -0.01 (95\% CI $[-1.11,1.09], p=0.98)$. Results are revealed in Fig. 3(e). Heterogeneity was high $\left(\mathrm{I}^{2}=91 \%\right.$; $\mathrm{H}^{2}=11.52$ ).

Visual inspection of funnel plot for publication bias obtained by the Rank Correlation Test for Funnel Plot Asymmetry proposed publication bias for $\mathrm{HFms}^{2}$ (in addition the non-significant Kendall's tau $=0.20$, $p=0.82)$.

\subsubsection{Frequency domain - LFnu}

Mendonça et al. (2013), Agiovlasitis et al. (2010) did not report the mean or $\mathrm{SD}$, and the data was obtained from the figure prior to analysis.

There was no significant difference in LFnu between the DS subjects and control group at rest, with outcomes presenting a weighted average effect size of -0.11 (95\% CI $[-2.13,1.92], p=0.92)$. Results are revealed in Fig. 3(f). Heterogeneity was high $\left(\mathrm{I}^{2}=95 \% ; \mathrm{H}^{2}=21.79\right)$.

Visual inspection of funnel plot for publication bias obtained by the Rank Correlation Test for Funnel Plot Asymmetry suggested publication bias for LFnu (also an insignificant Kendall's tau $=-1.00, p=0.33$ ).

\subsubsection{Frequency domain - HFnu}

Mendonça et al. (2013), Agiovlasitis et al. (2010) did not report the Mean or SD, and the datasets were obtained from the figure prior to analysis.

There was no significant difference in HFnu between the DS subjects and control group at rest, with outcomes showing a weighted average effect size of -0.15 (95\% CI $[-2.42,2.12], p=0.90)$. Results are displayed in Fig. 3(g). Heterogeneity was high $\left(\mathrm{I}^{2}=96 \% ; \mathrm{H}^{2}=25.89\right)$.

Visual inspection of funnel plot for publication bias obtained by the Rank Correlation Test for Funnel Plot Asymmetry suggested publication bias for HFnu (also a non-significant Kendall's tau $=0.33, p=1.00$ ).

\subsubsection{Frequency domain $-L F / H F$}

Agiovlasitis et al. (2010) did not report the Mean or SD, and the data were obtained from the figure before analysis.

There was no significant change in LF/HF between the DS subjects $(n=123)$ and control $(n=81)$ groups at rest, with outcomes showing a weighted average effect size of -0.94 (95\% CI $(-2.42,0.54)$, 


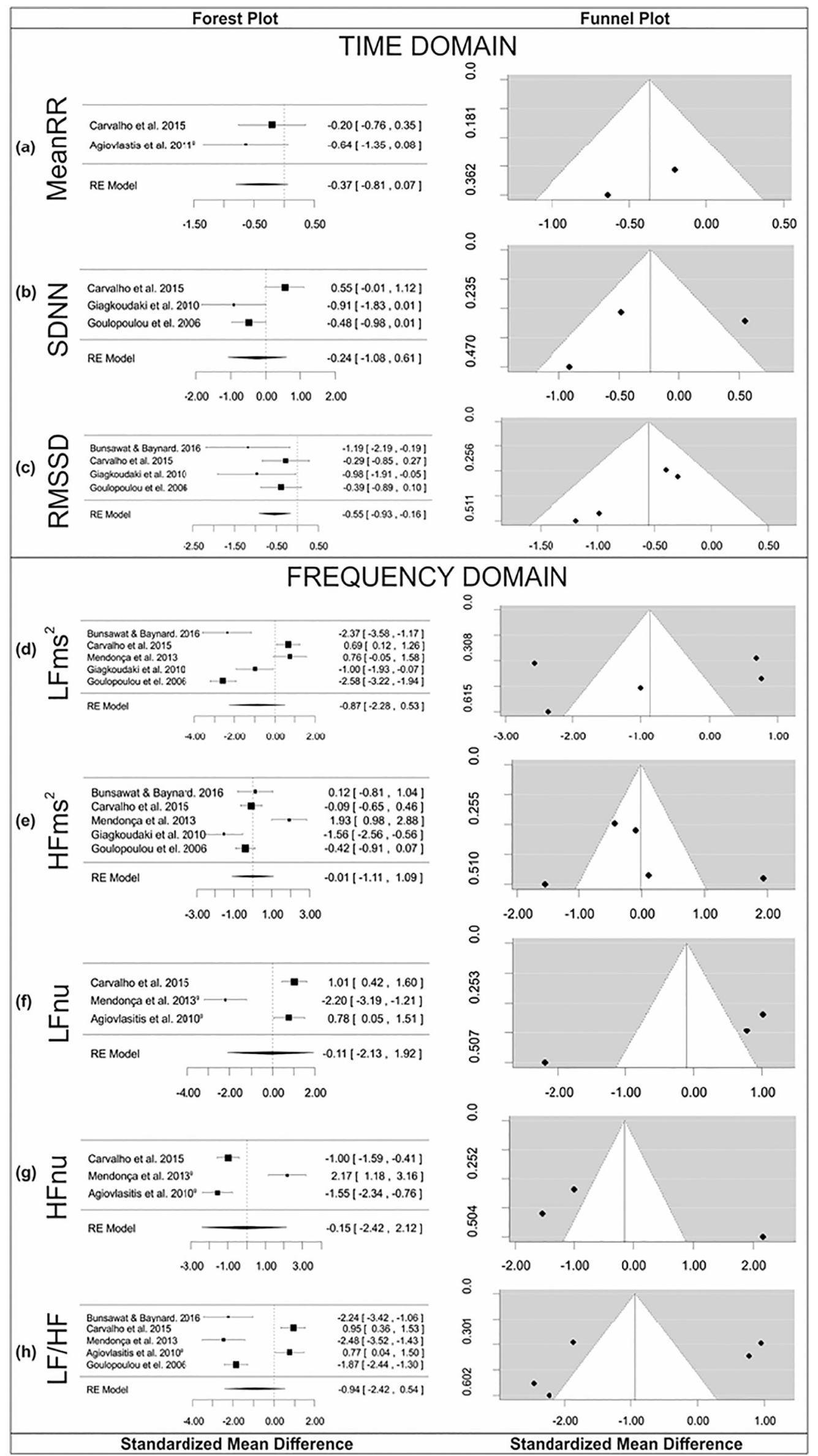


Fig. 3. Meta-analysis forest plot and funnel plot; results from random-effect meta-analysis comparing resting state MeanRR (a), SDNN (b), RMSSD (c), LFms ${ }^{2}$ (d), HFms $^{2}$ (e), LFnu (f), HFnu (g) and LF/HF (h) in subjects with DS and controls.

$p=0.21)$. Results are displayed in Fig. 3(h). Heterogeneity was high $\left(\mathrm{I}^{2}=95 \% ; \mathrm{H}^{2}=19.17\right)$.

Visual inspection of funnel plot for publication bias obtained by the Rank Correlation Test for Funnel Plot Asymmetry suggested publication bias for LF/HF (also a non-significant Kendall's tau $\leq 0.001, \mathrm{p}=1.00$ ).

\section{Discussion}

This study presents results of a systematic review and a meta-analysis about heart rate variability in individuals with DS. Therefore, we have chosen to organize the discussion in two parts: systematic review and meta-analysis.

\subsection{Systematic review}

Studies regarding cardiac autonomic modulation in DS are conducted with subjects from a wide age range, either gender, and no medications prescribed. Also, they presented HRV analysis at rest, in autonomic tests, exercise, and sleeping.

Isolated rest condition was evaluated in only one study (Carvalho et al., 2015), whose results indicated increasing indices representing the sympathetic branch of the ANS and those that indicate the overall modulation in children with DS.

Regarding autonomic tasks, some studies explore the autonomic response to upright tilt. In general, the results indicate that there is no difference between groups at rest, but, during postural change, DS individuals presented a significant decrease in Mean RR (Iellamo et al., 2005), reduced vagal withdrawal (Agiovlasitis et al., 2010), smaller increase in sympathetic modulation (Bunsawat et al., 2015; Agiovlasitis et al., 2010) and smaller decreases in HR complexity (Agiovlasitis et al., 2011) than individuals without DS.

Comparable autonomic responses have been observed in other autonomic tasks, such as isometric handgrip (Figueiroa Figueroa et al., 2005; Bunsawat and Baynard, 2016). Once more, the resting results were not different between groups and during handgrip and recovery; changes were greater in controls than in individuals with DS.

Regarding effect of physical exercise, despite the different methodologies (a session or in a multi-session program), the results are similar. Some studies (Baynard et al., 2004; Goulopoulou et al., 2006; Giagkoudaki et al., 2010) indicated differences in resting modulation between groups, and after exercise training, in the DS group there was an improvement of sympathovagal balance to levels seen in healthy individuals, chiefly by increasing vagal activity (Giagkoudaki et al., 2010; Mendonça et al., 2013).

Another condition evaluated in the studies is the sleeping condition and the conclusions were divergent. Ferri et al. (1998) observed an imbalance between the sympathetic and vagal systems with a predominant sympathetic activity in DS; and Sei et al. (1995) suggested that only in rapid eye movement sleep was there a distinct difference between the groups, sympathetic activity was lower in DS than in controls.

\subsection{Meta-analysis}

From studies that satisfied our selection criteria, only one (Angiovlasitis et al., 2011) performed the nonlinear analysis HRV, then our meta-analysis was performed only with linear indexes, comparing DS and controls during the resting state, present in all articles. Results from meta-analysis revealed that there was no significant difference between the groups at rest, except the RMSSD, which was lower in DS than controls.

As we have revealed, the information about cardiac regulation during the resting state in DS individuals diverges. Independent of their protocol, some studies have found no changes at rest (Iellamo et al., 2005; Figueiroa Figueroa et al., 2005; Angiovlasitis et al., 2011; Mendonça et al., 2013; Bunsawat et al., 2015); others describe an increase in the indices representing the sympathetic branch of the ANS (Carvalho et al., 2015), and others, greater parasympathetic activity at rest (Baynard et al., 2004). Lastly, there are those that indicated reduced total HRV at rest in DS (Giagkoudaki et al., 2010; Goulopoulou et al., 2006), in which there is the decrease in both branches of the ANS.

In these studies, with reduced overall modulation, there are cases where there is a specific decrease in vagal activity (Giagkoudaki et al., 2010; Mendonça et al., 2013), which has also been observed in our meta-analysis, since that RMSSD was lower in DS than controls and it expresses the parasympathetic branch of the ANS (Vanderlei et al., 2009; Arab et al., 2016). Assuming that parasympathetic and sympathetic nervous systems are the principal regulators of cardiac chronotropism and the key determinants of the magnitude of spontaneous cardiovascular variability (Task Force, 1996; Draghici and Taylor, 2016), the imbalance in their functioning could explain the chronotropic ineffectiveness typically reported in subjects with DS, even without congenital heart disease.

In general, when evaluating chronotropic incompetence, studies display that individuals with DS exhibit physiologically depressed autonomic responses to exercise and autonomic tasks, with lower sympathovagal balance. In relation to postural change, for example, the results indicated blunted sympathetic activation and vagal withdrawal associated with a lesser reduction in baroreflex (Iellamo et al., 2005; Agiovlasitis et al., 2010); and reduced sympathetic dominance (Bunsawat et al., 2015).

Similar findings have been observed in isometric handgrip, where individuals with DS had diminished HR and blood pressure responses due to blunted vagal response (Figueiroa Figueroa et al., 2005; Bunsawat et al., 2015); and during exercise. With regards to that, results have indicated that before an exercise program subjects with DS can exhibit impaired sympathovagal balance, with lesser vagal modulation, conceivably (Giagkoudaki et al., 2010); however, following exercise training, there is an improvement of sympathovagal balance to levels realized in healthy subjects, mainly by increasing vagal activity (Giagkoudaki et al., 2010; Mendonça et al., 2013).

Under regular conditions, during low to moderate intensity exercise, neural signals from the central command and muscle receptors evoke a decrease in vagal outflow, which sequentially mediates the increase in HR. Alongside vagal withdrawal, exercise induces increases in muscle nerve sympathetic activity (Task Force, 1996; Goulopoulou et al., 2006; Vanderlei et al., 2009). Despite similar resting autonomic control, as we have unveiled in our meta-analysis, and normal responses to sympathoexcitatory tasks (Figueiroa Figueroa et al., 2005; Bunsawat and Baynard, 2016), indications of the autonomic dysfunction in individuals with DS persist.

Although some researchers suggest that the problem is the parasympathetic decrease (Giagkoudaki et al., 2010; Mendonça et al., 2013), for others the dysfunction is sympathetic hyperactivity. For Carvalho et al. (2015), even though the individuals with DS respond physiologically to autonomic tasks, such responses are lower than that of controls, which could be explained by sympathetic predominance of these individuals, which hinder or delay the action of the vagus front of excitatory tasks (Carvalho et al., 2015).

Our review has revealed cases wherein differences between DS and controls present in resting state disappear with the onset of exercise, suggesting that other variables are responsible for chronotropic incompetence in persons with DS (Baynard et al., 2004), such as obesity. Undeniably, overweight and overfeeding require consideration in the 
supervision of persons with DS (Bull and Committee on Genetics, 2011; Van Gameren-Oosterom et al., 2012; Kazemi et al., 2016), since the prevalence of obesity in these individuals is around 31\% to $47 \%$ (Bull and Committee on Genetics, 2011; Bertapelli et al., 2016).

Considering that overweightness alone causes alterations in cardiac autonomic regulation (Vanderlei et al., 2010), that could contribute to autonomic dysfunction in the syndrome, as verified by Angiovlasitis et al. (2011), whose study exposed that the differences in HR complexity in response to passive upright tilt, observed in people with DS, was partially due to their higher BMI. Yet, there is also disagreement in the research literature, because some studies propose that autonomic dysfunction is independent of obesity in subjects with DS (Baynard et al., 2004; Mendonça et al., 2013; Bunsawat and Baynard, 2016).

In the study of Figueiroa Figueroa et al. (2005), monitoring of BMI did not modify the results and attenuated HR and SBP responses to handgrip exercise in individuals with DS remained associated with blunted vagal withdrawal. Goulopoulou et al. (2006) investigated the relationship between some variables and HRV, and BMI were not significantly correlated with resting cardiac autonomic control. These investigators suggested that obesity differentially affects autonomic control in subjects with DS compared to their non-disabled associates. Giagkoudaki et al. (2010) detected improvement of sympathovagal balance after exercise training, and their results displayed that neither body weight nor BMI affected cardiac autonomic regulation in subjects with DS.

Consistent with the reviewed studies, there is evidence for altered autonomic function in individuals with DS independently of the presence of cardiopathy. Yet, the exact mechanism leading to this dysfunction is not fully elucidated. Even though some studies have not met our selection criteria, we must emphasize that some hypotheses have been considered, such as muscular hypotonia (Kazemi et al., 2016; Kazemi et al., 2017), pathological abnormalities in DS brain (Sacks and Smith, 1989; Ferri et al., 1998; Van Gameren-Oosterom et al., 2012), and in neurotransmitters (Lake et al., 1979; Udeschini et al., 1985).

Muscular hypotonia is highly prevalent in these individuals (Bull and Committee on Genetics, 2011; Van Gameren-Oosterom et al., 2012; Kazemi et al., 2017) and could be an explanation for the reduced ability of cardiac autonomic response to demands during submaximal activities. This hypotonia could affect the cardiac muscle cells, with the potential to reduce the responsiveness to a certain level of vagal withdrawal and sympathetic activation, resulting in decreased absolute change in HR during exercise (Guerra et al., 2003; Fernhall and Otterstetter, 2003; Mendonça and Pereira, 2010; Fernhall et al., 2013).

Another option is that certain brain areas have been suggested as the source of autonomic dysfunction in DS (Sacks and Smith, 1989; Van Gameren-Oosterom et al., 2012). Some pathological abnormalities have been found in several areas of the DS brain, including the frontal cortex, hippocampus and cerebellum, along with diffuse cerebrocortical pathology that includes disorders in neuronal density, dendritic spine development and synaptic functioning (Dierssen et al., 1997).

In support of this conception, consistent findings of abnormalities in DS brains also indicate the most of them exhibit some evidence of Alzheimer's disease shortly after the age of 30 (Fernhall and Otterstetter, 2003). Plaques and tangles in the images appear 20 to 30 years earlier in DS and dementia associated with this disease and is clinically detected at least three times more frequently than in a control population. This would indicate signs of premature aging and all their panoply of consequences on the cardiovascular system (Sacks and Smith, 1989; Kazemi et al., 2016).

Finally, the observation of a revised balance between the sympathetic and vagal systems can be discussed regarding neurotransmitters. Some studies have revealed that DS patients had significantly higher circulating levels of norepinephrine while supine, standing and during cold pressor tests when compared to age-matched controls (Lake et al., 1979; Udeschini et al., 1985), suggesting that sympathetic tone is not reduced, but possibly exaggerated in DS (Ferri et al., 1998; Naveen and
Telles, 1999).

Taken together, our literature review and meta-analysis allow us to state that there is autonomic dysfunction in individuals with DS, which may or may not be expressed at rest, but it is usually demonstrated in autonomic tasks. As the precise mechanism of this autonomic imbalance is not yet fully understood, the analysis of HRV indexes can help us to characterize and monitor interventions and treatment for this population. Given the increase in their life expectancy, clinicians and scientists should direct their efforts to promote improvement of life quality of persons with DS.

This research presents several points that should be highlighted. Owing to the variability in the methodology of the selected studies, we have conducted the meta-analysis only with linear indexes, comparing DS and controls during the resting state, present in all articles. Still, it would not be possible to completely normalize the sample in relation to some characteristics. Overall, investigations are conducted with volunteers from a wide age range and with typical increases of BMI. Nevertheless, our meta-analysis included only studies whose subjects did not take medications, in the absence of physical activity and did not have cardiopathies.

Regarding the meta-analysis, the funnel plot assesses the hypothesis that the relationship between probability of variable studied and study size, measured by standard error, is independent. This was verified by a Kendall's tau, which were estimated with a $p$-value higher than 0.05 , suggesting there is no evidence of asymmetry. Although the presence of publication bias is a frequent explanation to an asymmetric funnel plot, data presented here are observational data without any intervention, so the funnel plot asymmetry could also be by reason of heterogeneity in the data (Ferguson, 2007; Sterne et al., 2011).

\section{Conclusion}

In summary, the literature review has revealed that there is autonomic dysfunction in individuals with DS, which may or may not be expressed at rest, but it is usually demonstrated in autonomic tasks. Secondly, meta-analysis indicated that there was no significant difference between the HRV indexes during rest, except RMSSD index which was lower in DS than control group.

\section{Declaration of interest}

All authors report no conflict of interest.

\section{Funding}

This work was supported by the FAPESP (Fundaçao de Amparo à Pesquisa do Estado de Sao Paulo), process number: 2010/18005-0.

\section{Acknowledgments}

We would like to thank all authors who were contacted and returned with information about their original data, especially to Tracy Barnard and her team from Integrative Physiology Laboratory, Department of Kinesiology and Nutrition, University of Illinois at Chicago, USA.

\section{References}

Agiovlasitis, S., Collier, S.R., Baynard, T., Echols, G.H., Goulopoulou, S., Figueroa, A., et al., 2010. Autonomic response to upright tilt in people with and without Down syndrome. Res. Dev. Disabil. 31, 857-863.

Angiovlasitis, S., Baynard, T., Pitetti, K.H., Fernhall, Bo, 2011. Heart rate complexity in response to upright tilt in persons with Down syndrome. Res. Dev. Disabil. 32, 2102-2107.

Arab, C., Dias, D.P., Barbosa, R.T., et al., 2016. Heart rate variability measure in breast cancer patients and survivors: a systematic review. Psychoneuro. Endocrinology 68, 57-68.

Baynard, T., Pitetti, K.H., Guerra, M., Fernhall, B., 2004. Heart rate variability at rest and 
during exercise in persons with Down syndrome. Arch. Phys. Med. Rehabil. 85, 1285-1290.

Bertapelli, F., Pitetti, K., Agiovlasitis, S., Guerra-Junior, G., 2016. Overweight and obesity in children and adolescents with Down syndrome: prevalence, determinants, consequences, and interventions: a literature review. Res. Dev. Disabil. 57, 181-192.

Bull, M.J., Committee on Genetics, 2011. Health supervision for children with Down syndrome. Pediatrics 128 (6), 1212.

Bunsawat, K., Baynard, T., 2016. Cardiac autonomic modulation and blood pressure responses to isometric handgrip and submaximal cycling exercise in individuals with Down syndrome. Clin. Auton. Res. 26 (4), 253-260.

Bunsawat, K., Goulopoulou, S., Collier, S.R., Figueroa, A., Pitetti, K.H., Baynard, T., 2015. Normal HR with tilt, yet autonomic dysfunction in persons with Down syndrome. Med. Sci. Sports Exerc. 47, 250-256.

Carvalho, T.D., Abreu, L.C., Mustacchi, Z., Vanderlei, L.C.M., Godoy, M.F., Raimundo, R.D., et al., 2015. Cardiac autonomic modulation of children with Down syndrome. Pediatr. Cardiol. 36, 344-349.

Dierssen, M., Vallina, I.F., Baamonde, C., Garcia-Calatayud, S., Lumbreras, M.A., Florez, J., 1997. Alterations of central noradrenergic transmission in Ts65Dn mouse, a model for Down syndrome. Brain Res. 749, 238-244.

Draghici, A.E., Taylor, J.A., 2016. The physiological basis and measurement of heart rate variability in humans. J. Physiol. Anthropol. 28 (35(1)), 22.

Ferguson, C.J., 2007. Evidence for publication bias in video game violence effects literature: a meta-analytic review. Aggress. Violent Behav. 12 (4), 470-482.

Fernhall, B., Otterstetter, M., 2003. Attenuated responses to sympathoexcitation in individuals with Down syndrome. J. Appl. Physiol. 94, 2158-2165.

Fernhall, B.O., Mendonca, G.V., Baynard, T., 2013. Reduced work capacity in individuals with Down syndrome: a consequence of autonomic dysfunction? Exerc. Sport Sci. Rev. 41 (3), 138-147.

Ferri, R., Dascalova, L.C., DelGracco, S., Elia, M., Musumeci, S.A., Pettinat, S., 1998. Heart rate variability and apnea during sleep in Down's syndrome. J. Sleep Res. 7, 282-287.

Figueiroa Figueroa, A., Collier, S., Baynard, T., Giannopoulou, I., Goulopoulou, S., Fernhall, B., 2005. Impaired vagal modulation of heart rate in individuals with Down syndrome. Clin. Auton. Res. 15, 45-50.

Giagkoudaki, F., Dimitros, E., Kouidi, E., Deligiannis, A., 2010. Effects of exercise training on heart-rate-variability indices in individuals with Down syndrome. J. Sport Rehabil. 19, 173-183.

Goulopoulou, S., Baynard, T., Collier, S., et al., 2006. Cardiac autonomic control in individuals with Down syndrome. Am. J. Ment. Retard. 111, 27-34.

Guerra, M., Llorens, N., Fernhall, B., 2003. Chronotropic incompetence in individuals with Down syndrome. Arch. Phys. Med. Rehabil. 84, 1604-1608.

Hutton, B., Salanti, G., Caldwell, D.M., et al., 2015. The PRISMA extension statement for reporting of systematic reviews incorporating network meta-analyses of health care interventions: checklist and explanations. Ann. Intern. Med. 162 (11), 777-784.

Iellamo, F., Galante, A., Legramante, J.M., Lippi, M.E., Condoluci, C., Albertini, Giorgio, Volterrani, M., 2005. Altered autonomic cardiac regulation in individuals with Down syndrome. Am. J. Physiol. Heart Circ. Physiol. 289, H2387-H2391.

Karim, N., Hasan, J.A., Ali, S.S., 2011. Heart rate variability - a review. Aust. J. Basic Appl. Sci. 7 (1), 71-77.

Kazemi, M., Salehi, M., Kheirollahi, M., 2016. Down syndrome: current status, challenges and future perspectives. Int. J. Mol. Cell. Med. 5 (3), 125-133.

Kazemi, M., Salehi, M., Kheirollahi, M., 2017. MeDIP real-time qPCR has the Potential for noninvasive prenatal screening of fetal trisomy 21. Int. J. Mol. Cell Med. Winter 6 (1), $13-21$.

Lake, C.R., Ziegler, M.G., Coleman, M., Kopin, I.J., 1979. Evaluation of the sympathetic nervous system in trisomy-21 (Down's syndrome). Psychiatry Res. 15, 1-6.

Massetti, T., da Silva, T.D., Ribeiro, D.C., et al., 2014. Motor learning through virtual reality in cerebral palsy-a literature review. In: MedicalExpress. 1. pp. 302-306.

Massetti, T., Trevizan, I.L., Arab, C., Favero, F.M., Ribeiro-Papa, D.C., de Mello Monteiro, C.B., 2016. Virtual reality in multiple sclerosis - a systematic review. In: Multiple Sclerosis and Related Disorders. 8. pp. 107-112.

Mendonça, G.V., Pereira, F.D., 2010. Heart rate recovery after exercise in adults with the Down syndrome. Am. J. Cardiol. 105, 1470-1473.

Mendonça, G.V., Pereira, F.D., Fernhall, B., 2013. Heart rate recovery and variability following combined aerobic and resistance exercise training in adults with and without Down syndrome. Res. Dev. Disabil. 34 (1), 353-361.

Menezes, L.D.C.D., Massetti, T., Oliveira, F.R., et al., 2015. Motor learning and virtual reality in Down syndrome; a literature review. Int. Arch. Med. 8, 1-11.

Naveen, K.V., Telles, S., 1999. Sudomotor sympathetic hypofunction in down's syndrome. Indian J. Physiol. Pharmacol. 43 (4), 463-466.

Peschel, S.K., Feeling, N.R., Vögele, C., Kaess, M., Thayer, J.F., Koenig, J., 2016. A metaanalysis on resting state high-frequency heart rate variability in bulimia nervosa. Eur. Eat. Disord. Rev. 24 (5), 355-365.

R Core Team, 2017. R: A Language and Environment for Statistical Computing. R Foundation for Statistical Computing, Vienna, Austria. http://www.r-project.org.

Sacks, B., Smith, S., 1989. People with Down's syndrome can be distinguished on the basis of cholinergic dysfunction. J. Neurol. Neurosurg. Psychiatry 52, 1294-1295.

Sei, H., Enai, T., Chang, H.Y., Morita, Y., 1995. Heart rate variability during sleep in Down's syndrome. Physiol. Behav. 58 (6), 1273-1276.

Sterne, J.A1., Sutton, A.J., Ioannidis, J.P., Terrin, N., Jones, D.R., Lau, J., et al., 2011. Recommendations for examining and interpreting funnel plot asymmetry in metaanalyses of randomised controlled trials. BMJ 22 (343), d4002.

Task Force of the European Society of Cardiology and the North American Society of Pacing and Electrophysiology, 1996. Heart rate variability: standards of measurement, physiological interpretation and clinical use. Circulation 93 (5), 1043-1065.

Udeschini, G., Casati, G., Bassani, F., Picotti, G.B., Culotta, P., 1985. Plasma catecholamines in Down's syndrome, at rest and during sympathetic stimulation. J. Neurol. Neurosurg. Psychiatry 48, 1060-1061.

Van Gameren-Oosterom, H.B.M., Van Dommelen, P., Oudesluys- Murphy, A.M., Buitendijk, S.E., Van Buuren, S., et al., 2012. Healthy growth in children with Down Syndrome. PLoS One 7 (2), e31079.

Vanderlei, L.C., Pastre, C.M., Hoshi, R.A., Carvalho, T.D., Godoy, M.F., 2009. Basic notions of heart rate variability and its clinical applicability. Rev. Bras. Cir. Cardiovasc. 24, 205-217.

Vanderlei, L.C.M., Pastre, C.M., Freitas Jr., I.F., Godoy, M.F., 2010. Analysis of cardiac autonomic modulation in obese and eutrophic children. Clinics 65 (8), 789-792.

Viechtbauer, W., Viechtbauer, M.W., 2016. Package 'metafor'. The Comprehensive R Archive Network. Package 'metafor'. Available in. https://cran.r-project.org/web/ packages/metafor/metafor.pdf. 